\title{
CONSIDERAÇÕES SOBRE A SEXUALIDADE E EDUCAÇÃO SEXUAL DE PESSOAS COM TRANSTORNO DO ESPECTRO AUTISTA ${ }^{1}$
}

\author{
CONSIDERACIONES SOBRE LA SEXUALIDAD Y EDUCACIÓN SEXUAL DE \\ PERSONAS CON TRANSTORNO DEL ESPECTRO AUTISTA
}

\author{
CONSIDERATIONS ON SEXUALITY AND SEXUAL EDUCATION OF PERSONS \\ WITH AUTISTIC SPECTRUM DISORDERS
}

\author{
Ana Carla Vieira OTTONI ${ }^{2}$ \\ Ana Claudia Bortolozzi MAIA ${ }^{3}$
}

RESUMO: O Transtorno do Espectro Autista (TEA) é um transtorno de desenvolvimento, cujas características principais são os déficits na comunicação social e os interesses ou comportamentos restritos e repetitivos. Este trabalho, focado nos níveis do TEA que exigem menos apoio, anteriormente descritos como Síndrome de Asperger e aqui denominados TEA/SA, apresenta uma revisão teórica sobre a sexualidade dessa população. Essa sexualidade é frequentemente invisibilizada, o direito de acesso à informação e a vivências sexuais comumente negados, e há vulnerabilidade com relação a abusos e violências. Os estudos indicam que os déficits em habilidades sociais, de comunicação, os interesses restritos e repetitivos e a hipersensibilidade são possíveis fatores limitantes do desenvolvimento sexual. Alguns dados apontam para a identificação de desejos sexuais voltados especialmente a vivências solitárias da sexualidade e menor frequência de práticas sexuais, em comparação à população como um todo. As dificuldades dos pais e profissionais em falar sobre sexualidade foram destacadas, e um programa eficiente de educação sexual para pessoas com TEA/SA foi apontado como um fator de proteção para um desenvolvimento satisfatório e para diminuição de vitimização, incluindo nessa intervenção aspectos importantes, como o uso de linguagem acessível, direta e clara; o ensino sistemático de habilidades sociais; a orientação familiar e profissional. Conclui-se que a revisão da literatura foi importante para esclarecer sobre a temática e subsidiar a elaboração de propostas de educação sexual para essa população, sem omitir, claro, os desejos e necessidades das próprias pessoas com TEA/SA.

PALAVRAS-CHAVE: Transtorno do espectro autista. Síndrome de Asperger. Sexualidade. Educação sexual.

RESUMEN: El trastorno del espectro autista (TEA) es un trastorno de desarrollo, cuyas características principales son los déficits en la comunicación social y los intereses o comportamientos restringidos y repetitivos. Este trabajo, enfocado en los niveles del TEA que

\footnotetext{
${ }^{1}$ Artigo referente a dados parciais da pesquisa de mestrado em Psicologia do Desenvolvimento e Aprendizagem na Faculdade de Ciências, Universidade Estadual Paulista "Julio de Mesquita Filho", UNESP/Bauru (VIEIRA, 2016), com apoio de Bolsa CAPES cedida pelo programa.

${ }^{2}$ Universidade Sagrado Coração (USC), Bauru - SP - Brasil. Docente no Centro de Ciências Humanas. Mestre em Psicologia. ORCID: <http://orcid.org/0000-0003-1112-3930>. E-mail: anacarlaunesp@gmail.com

${ }^{3}$ Universidade Estadual Paulista (UNESP), Bauru - SP - Brasil. Docente PPG Araraquara e Bauru, e na graduação em Psicologia. Doutora em Educação e Pós-doutora em Educação pela UNESP/Araraquara. ORCID: <http://orcid.org/0000-0003-4796-5451>.E-mail: aclaudia@fc.unesp.br
} 
requieren menos apoyo, anteriormente descritos como Síndrome de Asperger y aquí denominados TEA/SA, presenta una revisión teórica sobre la sexualidad de esa población. Esta sexualidad es frecuentemente invisibilizada, el derecho de acceso a la información y las vivencias sexuales comúnmente negadas, y hay vulnerabilidad con respecto a abusos y violencias. Los estudios indican que los déficits en habilidades sociales, de comunicación, los intereses restringidos y repetitivos y la hipersensibilidad son posibles factores limitantes del desarrollo sexual. Algunos datos apuntan a la identificación de deseos sexuales dirigidos especialmente a vivencias solitarias de la sexualidad y menor frecuencia de prácticas sexuales, en comparación a la población como un todo. Las dificultades de los padres y profesionales en hablar sobre sexualidad fueron destacadas, y un programa eficiente de educación sexual para personas con TEA/SA fue señalado como un factor de protección para un desarrollo satisfactorio y para disminuir la victimización, incluyendo en esa intervención aspectos importantes, como el uso de lenguaje accesible, directo y claro; la enseñanza sistemática de las habilidades sociales; la orientación familiar y profesional. Se concluye que la revisión de la literatura fue importante para esclarecer sobre la temática y subsidiar la elaboración de propuestas de educación sexual para esa población, sin omitir, claro, los deseos y necesidades de las propias personas con TEA/SA.

PALABRAS CLAVE: Trastorno del espectro autista. Síndrome de Asperger. Sexualidad. Educación sexual.

ABSTRACT: Autism Spectrum Disorder (ASD) is a developmental disorder, characterized by deficits in social communication and restricted/repetitive interests or behaviors. This work, focused on the levels of TEA that require less support, previously described as Asperger's Syndrome and here denominated TEA/SA, presents a theoretical revision on the sexuality of this population. Such sexuality is often invisible, the right of access to information and sexual experiences commonly denied, and there is vulnerability to abuse and violence. Studies indicate that deficits in social skills, communication, restricted and repetitive interests and hypersensitivity are possible limiting factors of sexual development. Some data point to the identification of sexual desires focused especially on solitary experiences of sexuality and less frequent sexual practices compared to the population. The difficulties of parents and professionals to talk about sexuality were highlighted, and an efficient program of sexual education for people with TEA/SA was appointed as a protective factor for a satisfactory development and reduction of victimization, including this intervention important aspects such as the use of accessible, direct and clear language; the systematic teaching of social skills; family and professional orientation. The literature review was important to clarify the issue and support the development of sexual education proposals for this population, without omitting, of course, the needs of the people with TEA/SA.

KEYWORDS: Autism spectrum disorder. Asperger Syndrome. Sexuality. Sexual education.

\section{Introdução}

Enquanto dimensão integrante dos seres humanos, a sexualidade deve fazer parte da elaboração de programas de inclusão que tenham como objetivo garantir os direitos da pessoa 
com deficiência em todas as esferas de sua vida. Os estudos, em geral, apontam para a escassez de programas educativos específicos sobre o assunto, para a privação de direitos e de vivências sexuais, para o pouco acesso a informações e, portanto, para a grande vulnerabilidade com relação à violência sexual (MAHONEY, 2011).

Dentre os fatores que contribuem para este quadro, Maia e Ribeiro (2010) citam os mitos e crenças atribuídos à sexualidade de pessoas com deficiência, como hipersexualidade ou assexualidade, incapacidade de obter parceiros, vínculos ou ter filhos, por exemplo. A desconstrução dessas ideias errôneas é essencial no movimento de elaboração de políticas e ações voltadas às necessidades reais dessas pessoas.

É necessário, ainda, evitar que sejam feitas generalizações a partir de limitações e desvantagens que determinada deficiência possa ocasionar, levando-as para outras áreas da vida da pessoa, como a afetiva e sexual (MAIA, 2009a). Atitudes como a infantilização de pessoas com deficiência, o foco em suas limitações e dependências e o destaque às características da condição em detrimento de aspectos pessoais individuais ou da fase do desenvolvimento também parecem contribuir para este quadro preocupante de invisibilidade (DANTAS; SILVA; CARVALHO, 2014; FRANÇA-RIBEIRO, 2004).

\section{Características do Transtorno do Espectro Autista (TEA)}

No contexto de estudo das deficiências, o Transtorno do Espectro Autista (TEA) tem sido foco de inúmeros trabalhos e movimentos sociais. Embora ainda incipientes, dados epidemiológicos recentemente citados por grandes instituições, como o Centro de Controle e Prevenção de Doenças norte-americano (CDCP), indicam números de prevalência de aproximadamente uma criança com TEA a cada cinquenta crianças (CENTERS FOR DISEASE CONTROL AND PREVENTION, 2013), evidenciando a necessidade de compreensão, estudo e tratamento sobre essa condição.

O TEA é descrito pelo Manual Diagnóstico e Estatístico de Transtornos Mentais (DSMV) a partir de duas categorias centrais: a) déficits persistentes na comunicação e na interação social em múltiplos contextos, como dificuldade para iniciar ou responder a interações sociais; déficits nos comportamentos comunicativos não verbais usados para interação social; problemas para desenvolver, manter e compreender relacionamentos; b) padrões restritos e repetitivos de comportamentos, interesses ou atividades, exemplificados por movimentos motores, uso de objetos ou fala estereotipados ou repetitivos; insistência nas mesmas coisas, 
adesão inflexível a rotinas ou padrões ritualizados de comportamento; hiper ou hiporreatividade a estímulos sensoriais ou interesse incomum por aspectos sensoriais do ambiente (AMERICAN PSYCHIATRIC ASSOCIATION, 2014).

Essas características devem sinalizar, na vida da pessoa, prejuízo em funcionamento social, profissional ou de outras áreas importantes da vida no momento da avaliação (AMERICAN PSYCHIATRIC ASSOCIATION, 2014). Uma peculiaridade importante do TEA é que os indivíduos manifestam os sintomas de maneiras radicalmente diferentes entre si (LAMPREIA, 2008), e para organizar os níveis de diagnósticos, há três classificações baseadas em suas necessidades: 1) exigindo apoio muito substancial; 2) exigindo apoio substancial; 3) exigindo apoio. O transtorno é diagnosticado quatro vezes mais frequentemente no sexo masculino que no feminino (AMERICAN PSYCHIATRIC ASSOCIATION, 2014).

Embora atualmente o conceito de TEA seja amplamente utilizado, antes da publicação do DSM-V os diagnósticos eram estabelecidos em subtipos do transtorno, como Transtorno Autista, Autismo de Alto Funcionamento, Autismo Atípico, Transtorno Desintegrativo da Infância e Síndrome de Asperger. As diferenças de características entre esses subtipos eram muito sutis, e a constatação da similaridade das condições forneceu embasamento para unificação nos termos do espectro (AMERICAN PSYCHIATRIC ASSOCIATION, 2014).

Perante a amplitude de características do transtorno, foi necessário que o presente estudo optasse por um nível específico do espectro, a fim de investigar sobre a sexualidade de sujeitos com características minimamente similares. O foco foi dado, portanto, em pessoas diagnosticadas com TEA no nível "exigindo apoio", ou com Síndrome de Asperger (SA), de acordo com os manuais psiquiátricos anteriores ao DSM-V. Para facilitar a compreensão, será o utilizado o termo TEA/SA com o objetivo de sinalizar referência a pessoas neste nível específico do espectro.

As pessoas com TEA/SA usualmente têm habilidades de inteligência e comunicação menos prejudicadas que nos outros níveis do espectro, sendo possível em alguns casos observar desempenhos inclusive acima da média (BRITO; NETO; AMARAL, 2013). Outra característica marcante é a dificuldade de convivência social, acentuada pelos déficits de compreensão da linguagem do outro - expressões faciais, metáforas, emoções e sinais.

Esses déficits se estendem, ainda, para a interpretação do que é ou não bem aceito socialmente, de mentiras ou situações potencialmente perigosas. Seus relacionamentos são frequentemente permeados pela dificuldade em expressar seus sentimentos e opiniões de forma assertiva e em aderir algumas regras sociais, como de vestimentas ou autocuidados (SEGAR, 2008). 
Outros estudos descritivos indicam características como o olhar nos olhos pouco frequente; o comportamento verbal erudito e formal; argumentações racionais; conversas focadas em objetivos específicos, sem muito envolvimento em comunicações informais. Pode haver também déficits para lidar com o inesperado ou com novidades, estereotipias e rituais cotidianos, ou o diagnóstico de Transtorno do Déficit de Atenção e Hiperatividade (TDAH) do subtipo desatento em comorbidade com o TEA/SA. Observa-se, muitas vezes, habilidades vantajosas, como ótima memória visual ou boa localização espacial (CAMARGOS Jr., 2013).

Um aspecto essencial descrito por Klin (2006) é o interesse e acúmulo de grande quantidade de informações sobre um tópico específico, que pode se alterar de tempos em tempos. Segar (2008) considerou que há casos nos quais os interesses duram a vida toda e outros, nos quais vão se alterando.

Acerca de prognósticos, há dificuldades para fazer previsões devido à imensa variabilidade de manifestações e características do TEA/SA, por isso estudos como o de Brito, Neto e Amaral (2013) indicam que o curso de vida depende muito das condições de desenvolvimento propiciadas. Em uma perspectiva que considera a deficiência como um fenômeno multidimensionado, ou seja, reconhece que há aspectos econômicos, culturais, sociais, de gênero, de raça implicados nas expressões de desvantagens sociais, é importante refletir sobre como o TEA/SA é diferente também a partir do nível de apoio e adaptações sociais encontrados pela pessoa.

\section{O processo de inclusão para pessoas com TEA/SA}

Em uma perspectiva de inclusão social, a sociedade deve intervir nos casos de pessoas com deficiência de forma a fornecer os suportes necessários para que elas acessem seus direitos, espaços e recursos da comunidade: econômicos, sociais, instrumentais, arquitetônicos, educativos, etc. Essa visão, denominada "Paradigma de Suporte", prevê que deve haver mudanças no modo como a sociedade se organiza e recebe a pessoa com deficiência e, simultaneamente, existir investimento no desenvolvimento de seus potenciais individuais (ARANHA, 2001). Dizendo de outro modo, são as modificações sociais que devem acolher às diferenças e não "mudar" individualmente o sujeito para adaptar-se à sociedade. É o que chamamos "modelo social" da deficiência (MAIA, 2011).

No contexto da educação sexual, por exemplo, essa visão poderia ser aplicada de forma ampla nas modificações sociais como desconstrução do preconceito, dos mitos e crenças sobre 
a sexualidade de pessoas com deficiência, e de forma focal em um programa de ensino sobre sexualidade com adaptações a partir das necessidades individuais.

O TEA/SA deve ser visto como um conjunto de características em interação com o contexto no qual o sujeito está inserido e esse reconhecimento fez com que fosse considerado "deficiência" no Brasil por meio da Lei 12764/2012. Este foi um acontecimento tardio, considerando-se que o autismo vem sendo discutido desde a década de 1940, mas que passou a oferecer subsídios em termos de direitos para a inclusão social dessa população. França-Ribeiro (2004) reconhece que a existência de documentos oficiais não é suficiente para mudanças nas práticas, porém é importante apontar que podem servir de instrumentos de acesso.

A Lei Brasileira de Inclusão (LBI), aprovada em 2015, descreve direitos das pessoas com deficiência em múltiplos contextos incluindo aspectos importantes relacionados à sexualidade da pessoa com deficiência. $\mathrm{O}$ artigo sexto, especificamente, indica que:

\begin{abstract}
A deficiência não afeta a plena capacidade civil da pessoa, inclusive para casar-se e constituir união estável; exercer direitos sexuais e reprodutivos; exercer o direito de decidir sobre o número de filhos e de ter acesso a informações adequadas sobre reprodução e planejamento familiar; conservar sua fertilidade, sendo vedada a esterilização compulsória; exercer o direito à família e à convivência familiar e comunitária e exercer o direito à guarda, à tutela, à curatela e à adoção, como adotante ou adotando, em igualdade de oportunidades com as demais pessoas (BRASIL, 2015).
\end{abstract}

Além disso, a curatela, ou atribuição a algum adulto da proteção e administração de bens e direitos civis da pessoa com deficiência, passou a ser, a partir da LBI, uma medida excepcional. $\mathrm{O}$ adulto que receber a função de cuidador afeta somente atos relacionados aos direitos de natureza patrimonial e negocial, não alcançando o direito ao corpo, à sexualidade, ao matrimônio, à privacidade, à educação, à saúde, ao trabalho e ao voto (BRASIL, 2015).

Para que as pessoas com deficiência tenham oportunidade de acesso a seus direitos sobre sexualidade, é importante:

[...] investir na educação sexual destas pessoas, tanto na família quando nas instituições. É preciso também orientar, e oportunizar a essas pessoas exteriorizarem desejos, afetos, dúvidas, medos, etc., para que eles aprendam a manifestar adequadamente esses sentimentos e, se possível, efetivar relações sexuais saudáveis e com responsabilidade, prevenindo-se de doenças, abusos sexuais e violência (MAIA, 2009b, p. 144).

Esse processo de educação sexual pode ocorrer formalmente, de maneira planejada e organizada com essa finalidade, por meio de programas de intervenção ou aulas e cursos, ou informalmente, como no caso dos valores, regras e concepções que existem nos discursos 
familiares, religiosos, midiáticos etc. (MAIA, 2011). Para elaborar programas e intervenções em educação sexual, voltados a uma população específica, como no caso de pessoas com TEA/SA, é importante basear-se em dados empíricos e validar a contribuição científica sobre a expressão da sexualidade nesses casos e/ou o sucesso ou não das experiências já realizadas.

\section{A sexualidade de pessoas com TEA/SA}

Mary e Jerry Newport foram diagnosticados com TEA/SA ainda jovens e, quando adultos, escreveram um livro sobre seu desenvolvimento sexual e afetivo. O casal utiliza a seguinte analogia para iniciar a descrição de sua experiência:

[a sexualidade] é uma importante dança de desenvolvimento para todos os jovens. É uma dança que grande parte das crianças autistas nem mesmo percebe até que seja óbvio para todo mundo já há bastante tempo. Então, eles querem dançar, mas perderam anos de aulas de pré-dança (NEWPORT; NEWPORT, 2002, p. 1).

Eles começam citando que a vivência da sexualidade se constrói ao longo do tempo por meio de comportamentos pré-requisitos, que não é algo "natural”, nem um acontecimento repentino na vida do adolescente. Este é um primeiro ponto essencial de análise: a concepção errônea de que comportamentos simplesmente surgem no repertório de crianças e adolescentes sem que seja necessário ensiná-los formalmente.

Em nossa sociedade, devido ao fato de a sexualidade representar um tabu e haver poucos programas dispostos a discuti-la com crianças e adolescentes, os adultos a tratam como se seu aprendizado fosse "natural" e espontâneo. Dessa forma, os adolescentes aprendem sobre sexualidade observando seus pares, questionando e buscando informações em espaços como a internet. Para a pessoa com TEA/SA, que tem dificuldades em interações sociais, interpretação de figuras de linguagem e sinais sutis, este tipo de educação sexual informal não é eficaz.

Neste sentido, Natale e Oliveira (2013) comentam que o ensino sobre comportamentos relacionados à sexualidade seria mais efetivo se fosse estruturado, assim como Segar (2008), que afirma:

A coisa mais difícil em ser autista (ou ter Síndrome de Asperger) é que as pessoas esperam que você conheça regras e viva de acordo com elas tal como elas fazem, apesar de ninguém nunca ter dito nada a você sobre elas. Não há dúvida de que isso é extremamente injusto, mas infelizmente a maioria das pessoas não verá desta maneira, porque elas não entendem o problema (SEGAR, 2008, p.11). 
Um segundo aspecto essencial na análise da sexualidade de pessoas com TEA/SA é o treino de habilidades sociais enquanto um fator de proteção. Para Newport e Newport (2002), sem um treinamento social intenso em crianças pequenas, elas não podem estar prontas para participar das interações sociais do período da puberdade, seja em situações sexuais e afetivas ou no contexto de amizades e grupo identitário. Um exemplo utilizado foi o da hipersinceridade: muitas vezes, a pessoa com TEA/SA emite uma opinião, por exemplo, dizendo "Você não está bonito hoje", e não consegue entender porque gerou chateação. A habilidade social de empatia e compreensão do ponto de vista do outro poderia, portanto, favorecer os vínculos de seus relacionamentos.

Ainda segundo os autores acima, outras habilidades que precisam ser treinadas, como pré-requisitos para a vivência da sexualidade, são: iniciar interação social; dosar o uso do humor; habituar-se melhor às mudanças inesperadas, pois o fato de não lidarem bem com elas pode gerar incompatibilidades na convivência cotidiana; desenvolver um equilíbrio entre o falar e o ouvir, compreendendo que seu interesse por assuntos específicos pode não ser compartilhado por outros, etc. (NEWPORT; NEWPORT, 2002).

Stokes, Newton e Kaur (2007) também indicaram que muitos comportamentos como iniciar de forma inadequada uma aproximação, tocar a pessoa de forma invasiva, acreditar que seu alvo deve corresponder a seus sentimentos e fazer comentários impróprios foram mais descritos pelas pessoas com TEA/SA que por aquelas com desenvolvimento típico. Segundo os autores, as pessoas com TEA/SA podem não conseguir moderar suas expressões e serem considerados perseguidores, além de apresentarem maior insistência frente a uma resposta negativa. Devido a essas dificuldades de interpretação das normas sociais, muitas pessoas com TEA acabam por serem vistas como "chatas" ou "incômodas" e em alguns casos, essas questões poderiam, até mesmo, tomar dimensões mais drásticas, com ações legais e judiciárias (STOKES; NEWTON; KAUR, 2007).

Todas essas contribuições são essenciais para que, em um programa de educação sexual voltado a essa população, sejam previstas intervenções como o Treinamento de Habilidades Sociais (THS), especificamente a partir da perspectiva da Análise Aplicada do Comportamento, cuja eficácia vem sendo descrita por diversos estudos científicos (CAMARGO; RISPOLI, 2013). Kalyva (2010) também afirma a necessidade de investir na prática do treino de regras sociais e Teodoro, Casarini e Scorsolini-Comin (2013) falam sobre o desenvolvimento da comunicação social, como treino de compreensão não literal e contato visual.

Com relação aos comportamentos sexuais, a maior parte dos estudos indica que pessoas com TEA/SA se engajam mais em comportamentos solitários. Hellemans et al. (2007) 
identificaram que as habilidades sócio-sexuais eram deficitárias para grande parte dos participantes com TEA/SA de seu estudo, como em aspectos de higiene íntima, toques públicos inadequados e de comunicação. Gilmour, Schalamon e Smith (2012) concluíram que não havia razão a priori para sugerir que os indivíduos com TEA não tinham interesses e comportamentos sexuais, no entanto faltava investimento no desenvolvimento de programas em educação apropriados para esta população. $46 \%$ dos participantes de seu estudo afirmaram dirigir carícias a outras pessoas; $17 \%$ não se importavam com isso; $21 \%$ de vez em quando beijavam outras pessoas e $8 \%$ não se atentavam ao fato de o par gostar ou não do contato.

Há também nos relatos de vários autores, a preocupação sobre essas pessoas a se tornarem vítimas de abusos sexuais. Newport e Newport (2002) relataram a experiência da autora, Mary, durante a adolescência, pois a mesma vivenciava a necessidade de aceitação e popularidade e percebeu nas relações sexuais uma oportunidade de obter isso. Assim, ela viveu experiências abusivas que representaram, como ela mesma descreveu, uma das partes mais tristes de sua vida.

Camargos Jr. e Teixeira (2013) também abordaram este assunto, dizendo que a ingenuidade das pessoas com TEA/SA poderia trazer prejuízos como bullying ou violências sexuais, pois seria muito difícil para elas entender as diferenças entre interesses sexuais, afetivos, etc. Neste mesmo sentido, Kalyva (2010) disse que as sutilezas envolvidas na sexualidade, nos comportamentos sexuais e os déficits para compreender as expectativas sociais, poderiam fazer com que se tornassem vítimas ou atacadores. Ballan (2012) cita que outro fator de risco para essa situação é o fato de muitos pais de seu estudo demonstraram não vislumbrar no futuro de seus/as filhos/as o envolvimento em relações sexuais, resultando em falta de atenção à saúde sexual por parte de cuidadores.

Outras pesquisas endossam a discussão sobre a vulnerabilidade de pessoas com TEA em sofrerem situações de violência. Fisher, Moskowitz e Hodapp (2013) concluíram pessoas com TEA têm menos proteção social de pares e poucos amigos, além de serem mais propensos a se tornarem vítimas por meio do bullying, abuso sexual ou abuso físico devido às dificuldades em interpretar as intenções de outras pessoas. Em concordância com este dado, Sevlever, Roth e Gillis (2013), comentam que as características do transtorno colocam os indivíduos em situação de risco para abusos sexuais e que a educação sexual seria a ferramenta necessária como proteção, servindo para ajudá-los em seu desenvolvimento, minimizando o risco de ser explorado ou explorar alguém sexualmente.

Dentre as preocupações expressas pelas pessoas com TEA/SA sobre sua sexualidade, Mehzabin e Stokes (2011) indicam que eles/as relatam sentimentos de angústias quanto a seus 
próprios futuros, principalmente por temerem serem mal interpretados em seus comportamentos sexuais, ou ainda, quando temem não conseguirem conquistar um/a parceiro/a amoroso. Já na pesquisa de Hellemans et al. (2007), os participantes com TEA indicaram vontade de se relacionar com alguém, especialmente devido ao desejo de tentarem ser iguais às pessoas neurotípicas jovens, de terem afetividade, de fazerem coisas juntos, e para terem uma pessoa para cuidar de si. $54 \%$ dos sujeitos já havia mantido um relacionamento amoroso ao menos uma vez, mas desses, somente $13 \%$ tiveram uma relação sexual, o restante estava relacionado a andar de mãos dadas, tocar e beijar.

Com relação à visão dos familiares sobre o assunto, ou seja, dos primeiros e principais educadores sexuais dessa população, a preocupação maior, segundo Amaral (2009), diz respeito à ocorrência do abuso sexual e das mudanças do corpo que viriam com o tempo. A autora identificou, ainda, a percepção do adolescente como um bebê, com intensa infantilização do/a filho/a; esperanças de que um dia ele chegasse a namorar e oscilações entre a negação e a esperança acerca do desenvolvimento sexual.

Os familiares participantes da pesquisa de Ballan (2012) indicaram preocupação sobre os comportamentos sexuais e não sexuais de seus/suas filhos/as serem confundidos e estigmatizados por outras pessoas. Na busca por mecanismos que pudessem minimizar comportamentos não aceitos socialmente, alguns familiares aderiram a intervenções como colar pela casa papéis vermelhos onde não podia ficar sem roupa e papel verde onde podia e outros utilizaram o espaço de terapias para ensinar essas habilidades, recursos importantes como estratégias pedagógicas que podem ser usados em casa, mas também em escolas e em outros contextos.

Em geral, os pais expressaram achar muito difícil imaginar seus filhos com pares no futuro, disseram que os/as filhos/as não tinham amigos, por isso um/a parceiro/a ou namorado/a parecia improvável. Baseados em suas experiências, os pais relataram que precisavam preparar os filhos para as possíveis frustrações trazidas pelas paqueras, para que as negações que pudessem receber ou frustrações vivenciadas não afetassem a autoestima. Outras preocupações, que pareceram dificultar a expectativa dos filhos arranjarem pares, foram: a hipersensibilidade a cheiros ou as dificuldades nas interações sociais e nas emoções sentidas, comum entre a população com TEA (BALLAN, 2012). 


\section{Educação Sexual para pessoas com TEA/SA}

Dentre os estudos apresentados, muitos propõem intervenções com características, objetivos e pressupostos variados para atuar sobre a sexualidade de pessoas com TEA/SA. Para Mehzabin e Stokes (2011), por exemplo, é urgente a necessidade de programas especializados em educação sexual com a incorporação de questões como regras sociais, comunicação e compreensão e para Hellemans et al. (2007) o ensino da masturbação também pode ser necessário, citando como possíveis recursos, as instruções verbais, as fotografias ou vídeos. Stokes, Newton e Kaur (2007) ressaltam a importância dos relacionamentos com pares e amigos para o desenvolvimento das habilidades sociais e para o favorecimento de relacionamentos amorosos e sexuais.

Fisher, Moskowitz e Hodapp (2013) afirmam que o estabelecimento de uma forte rede de relacionamentos parece prioridade no programa de diminuição de vulnerabilidade da população com TEA/SA, pois o isolamento social aumenta a vitimização. A promoção de habilidades sociais para que estabeleçam relacionamentos também pode se tornar, na opinião dos autores, um fator de proteção.

Para Maia et al. (2015) a formação continuada de educadores é importante para que eles/as assumam e desenvolvam a educação sexual no contexto escolar para todos os/as alunos/as e, Vieira e Maia (2013) lembram que isso se aplica também aos alunos/as com deficiências em geral. Moreira (2007) indica o trabalho em grupo como uma intervenção interessante para a educação sexual de pessoas com deficiência, citando ideias de dinâmicas e oficinas que podem ser muito úteis. Koller (2000) cita que o ideal é que as intervenções sejam: mais concretas que abstratas; breves, específicas e claras; visuais; utilizar imitações e roleplayings; realizadas em situações de vida real e repetidas frequentemente.

França-Ribeiro (2004) diz que nessa fase de transição para a vida adulta e de busca pela independência, os serviços de ajuda devem ser oferecidos por meio de programas de educação sexual, de aconselhamento ou de terapia sexual. Ele comenta também que em alguns países há o incentivo da independência da pessoa com deficiência, de forma que os adultos têm seus apartamentos, moram sozinhos ou em grupos, tendo namorados/as caso desejem, e espaços privados para vivência da intimidade. Pode haver, em casos de necessidades, suportes ocasionais e ajuda para a gestão do cotidiano da moradia, o que sabemos ser algo ainda complexo na realidade brasileira.

Para Maia (2009b) o importante é que os profissionais que trabalham com pessoas com deficiência não limitem sua visão aos estereótipos e preconceitos, não reproduzam concepções 
pessoais e reflitam continuamente sobre suas ideias e atitudes. Ações essenciais, em sua opinião, estão no apoio para construção de autoimagem e autoestima na fase de transição entre infância e vida adulta. Dantas, Silva e Carvalho (2014) defendem que as intervenções devem ser realizadas para o empoderamento, para o conhecimento da auto-advocacia e dos direitos sexuais como possibilidades de abrir caminhos para o protagonismo na educação, no trabalho, na vida familiar, afetiva e sexual. Isso implica mudar o foco da deficiência para as habilidades, combatendo a cultura da incapacitação da pessoa com deficiência e da sua desvalorização.

Para Aston (2012) uma possibilidade de trabalho seria com foco em questões que podem surgir quando um ou ambos parceiros/as têm TEA/SA e procuram terapeutas para auxiliá-los. Para isso, ela afirma, é necessário que o profissional entenda sobre o transtorno e consiga trabalhar com a díade conjuntamente, auxiliando-o a atingir níveis de intimidade, sem enquadrar de antemão a pessoa no estereótipo do TEA/SA, compreendendo suas necessidades específicas.

Dekker et al. (2015) desenvolveram o Tackling Teenage Training (TTT) como programa para educação sexual de pessoas com TEA/SA. O treinamento leva seis meses e se inicia com uma reunião introdutória para que os pais e adolescentes conheçam o programa. A aplicação individual foi composta por 18 sessões cobrindo os seguintes tópicos: discussão sobre a puberdade; aparência; primeiras impressões; desenvolvimento físico e emocional na puberdade; como fazer amigos e manter amizades; se apaixonando e tendo um encontro; sexualidade e sexo; orientação sexual, masturbação e relação sexual segura; gravidez; estabelecendo e respeitando limites; uso da internet. Seu objetivo principal é aumentar o nível de conhecimento sobre sexualidade para possivelmente obter melhor funcionamento sexual.

Neste estudo, as sessões são estruturadas da seguinte forma: os adolescentes recebem informações sobre os tópicos e depois fazem exercícios sobre eles. Em cada sessão há folhetos com as informações e os exercícios, ancorados em ilustrações e um kit de treino com objetos como um pênis-modelo e camisinhas. Ao final de cada sessão é entregue uma tarefa para casa, de modo que eles possam praticar em outro contexto aquele conhecimento e os pais são informados via e-mail sobre o conteúdo da sessão anterior, assim como particularidades necessárias. Esse feedback dos pais é realizado com autorização dos filhos. O conhecimento dos adolescentes em aspectos psicossociais da sexualidade aumentou significativamente. Os pais relataram perceber uma generalização dos conhecimentos da situação de ensino para as situações cotidianas (DEKKER et al., 2015).

Outro tipo de intervenção interessante proposta para a população com TEA/SA é o uso de histórias sociais para ensinar habilidades ou conteúdos acerca da sexualidade, comentado 
por Tarnai e Wolfe (2008). Em geral, essas histórias são escritas na primeira pessoa do singular e as respostas ou comportamentos devem ser sempre utilizados no formato positivo, como "eu vou usar esse tom de voz" ao invés de "não devo falar alto". As histórias sociais têm basicamente seis sentenças com a seguinte estrutura: descrição da situação social em que aquela habilidade será importante; do comportamento adequado; dos sentimentos ou respostas da outra pessoa nessa situação; expressão de um valor ou opinião social sobre aquela situação; ensino sobre como e onde identificar, quando usar este comportamento e descrição do que outras pessoas farão para ajudar o estudante (TARNAI; WOLFE, 2008).

Em uma crítica sobre as pesquisas conduzidas acerca da sexualidade de pessoas com deficiências, Rosqvist (2014) comenta que as mesmas têm interpretado essa sexualidade como um déficit, algo destoante do que as pessoas neurotípicas apresentam, mas o movimento da defesa da auto-advocacia vem produzindo materiais em outra perspectiva. A revista "Empowerment", por exemplo, publica a partir da reivindicação da construção da identidade sexual nos próprios termos da pessoa com deficiência sem tomar como parâmetros as habilidades e características neurotípicas, assim como direito de estabelecer relacionamentos como quiserem.

Esse discurso de auto-advocacia busca, portanto, incluir a sexualidade como uma categoria a ser analisada dentre os indivíduos desta comunidade e não em comparação aos ditos sem deficiência, evidenciando a necessidade da ciência abrir espaço e dar voz efetivamente, às próprias pessoas com TEA: o que pensam e desejam sobre sua própria sexualidade.

\section{Considerações finais}

A análise de artigos a partir de uma revisão teórica sobre a sexualidade de pessoas com TEA/SA foi apresentada de modo organizado visando contribuir para a elaboração de programas e intervenções baseados em dados apontados pela literatura. Os diversos estudos convergem em algumas temáticas importantes: a) características da sexualidade no desenvolvimento de pessoas com TEA/SA; b) dificuldades nos relacionamentos amorosos e nas práticas sexuais; c) maior vulnerabilidade a violências e d) assexualidade na concepção de familiares.

Um primeiro dado enfatizado por diversos autores foram os possíveis fatores limitantes comuns no desenvolvimento sexual de pessoas com TEA/SA, tais como: dificuldades em desenvolver habilidades sociais favoráveis, dificuldades de comunicação, os interesses restritos 
e repetitivos e a hipersensibilidade (MEHZABIN; STOKES, 2011; NATALE; OLIVEIRA, 2012; NEWPORT; NEWPORT, 2002; SEGAR, 2008; STOKES; NEWTON; KAUR, 2007).

No campo dos relacionamentos amorosos e sociais, há destaque para os receios dos comportamentos de outras pessoas (sexuais ou afetivos) serem mal interpretados (MEHZABIN; STOKES, 2011; NEWPORT; NEWPORT, 2002) e dificuldades de comunicação e habilidades interpessoais. Quanto à vivência sexual, vários estudos ressaltam a identificação de desejos sexuais, porém realizados, em geral, com autoerotismo (BYERS; NICHOLS; VOYER, 2013; DEWINTER et al., 2015; HELLEMANS et al., 2007; KALYVA, 2010; NATALE; OLIVEIRA, 2013; OUSLEY; MESIBOV, 1991; TISSOT, 2009) ou com práticas sexuais com parceiros/as, porém em menor frequência em comparação à população como um todo (STOKES; KAUR, 2005).

A vulnerabilidade a situações de violências entre as pessoas com TEA/SA aparece em muitos estudos, ressaltando as ocorrências de abusos sexuais, violências e bullying (BALLAN, 2012; BROWN-LAVOIE; VIECILI; WEISS, 2014; CAMARGOS JR.; TEIXEIRA, 2013; FISHER; MOSKOWITZ; HODAPP, 2013; KALYVA, 2010; NEWPORT; NEWPORT, 2002, SEVLEVER; ROTH; GILLIS, 2013; SRECKOVIC; BRUNSTING; ABLE, 2014) indicando a necessidade de atenção para o assunto por parte dos profissionais, educadores e familiares envolvidos.

Estudos também apontaram as concepções de familiares sobre uma assexualidade de seus filhos/as com TEA/SA, evidenciando dificuldades dos adultos em reconhecê-los como seres sexuados e/ou em dialogar sobre isso (AMARAL, 2009; BALLAN, 2012; NEWPORT; NEWPORT, 2002). Devido aos dados relacionados às dificuldades da família com relação à sexualidade do/a filho/a com TEA/SA, um programa abrangente de educação sexual poderia acolher suas demandas específicas e orientar os membros familiares, discutindo possibilidades de ações educativas em casa. Da mesma forma, a formação de profissionais como professores e psicólogos da área parece essencial.

Apesar dos artigos não relacionarem, especificamente, a análise de programas de educação sexual institucionais, as questões levantadas em todos eles culminam para a compreensão de que um programa eficiente de educação sexual para pessoas com TEA/SA pode ser um fator de proteção para um desenvolvimento satisfatório e para diminuição de vitimização com relação às violências e, basicamente, as habilidades comportamentais devem valer-se de um ensino sistematizado que, no caso das pessoas com TEA/SA são: comunicação assertiva, empatia, identificação de sinais emitidos pelo outro, compreensão da linguagem não verbal e de situações sutis como paquera ou possíveis violências. 
Uma característica importante a ser considerada nessa elaboração, a partir dos dados encontrados, é o uso de linguagem acessível, ou seja, direta e clara, dada a frequente dificuldade de compreensão abstrata e de figuras de linguagem dessas pessoas, ou seja, considerar as características específicas nas pessoas que têm TEA/SA, para que elas recebam adequadamente as informações e treino de habilidades necessárias. Parte-se das habilidades e déficits dos aprendizes, utiliza-se de materiais acessíveis e que atendam às suas necessidades, procede-se avaliação processual e as generalizações das habilidades adquiridas a longo prazo. Nesse sentido, podem ser necessárias adaptações de diversas naturezas, a depender do repertório do participante e, claro, considerar a orientação necessária das pessoas que fazem parte do seu contexto social.

Finalmente, os estudos da área da neurodiversidade e auto-advocacia reivindicam o direito das pessoas com deficiência falarem sobre sua própria sexualidade e participarem da construção de políticas e ações sobre o assunto, que dizem respeito a si mesmas. Em uma perspectiva de representatividade esta é uma demanda justa, portanto é importante que os programas sejam elaborados também a partir de suas necessidades expressas e desejos revelados.

Conclui-se que a revisão da literatura realizada foi importante para esclarecer essa importante temática e subsidiar a elaboração de propostas de educação sexual para essa população que, frequentemente tem sua sexualidade invisibilizada; seus direitos de acesso à educação sexual de qualidade e às vivências sexuais comumente negados e, além de serem, especialmente, vulneráveis às situações de violências.

\section{REFERÊNCIAS}

AMARAL, C. E. S. O reconhecimento dos pais sobre a sexualidade dos filhos adolescentes com autismo e sua relação com a coparentalidade. 2009. 85 f. Dissertação (Mestrado em Psicologia) - Instituto de Psicologia, Universidade Federal do Rio Grande do Sul, Porto Alegre, 2009.

AMERICAN PSYCHIATRIC ASSOCIATION. Manual Diagnóstico e Estatístico de Transtornos Mentais. 5 ed. Porto Alegre: ARTMED, 2014.

ANDRADE, A. A.; TEODORO, M. L. M A. Implicações do Transtorno do Espectro do Autismo de Alto Funcionamento na dinâmica familiar. In: CAMARGOS JR., W. Síndrome de Asperger e outros Transtornos do Espectro do Autismo de Alto Funcionamento: da avaliação ao tratamento. Belo Horizonte: Artesã, p. 197-212, 2013.

ARANHA, M. S. F. Paradigmas da relação da sociedade com as pessoas com deficiência. Revista do Ministério Público do Trabalho, n. 21, p. 160-173, 2001. 
ASTON, M. Asperger syndrome in the bedroom. Sexual and Relationship Therapy, v. 27, n. 1, p. 73-79, 2012.

BALLAN, M. S. Parental Perspectives of Communication about Sexuality in Families of Children with Autism Spectrum Disorders. Journal of Autism Developmental Disorders, v. 42, p. 676-684, 2012.

BRASIL. Lei ${ }^{\circ} 13.146$ de 06 de julho de 2015. Institui a Lei Brasileira de Inclusão da Pessoa com Deficiência (Estatuto da Pessoa com Deficiência). Brasília: 2015. Disponível em http://www.planalto.gov.br/ccivil_03/_ato2015-2018/2015/lei/113146.htm. Data de acesso: jul. 2017.

BROWN-LAVOIE, S. M.; VIECILI, M. A.; WEISS, J. A. Sexual Knowledge and Victimization in Adults with Autism Spectrum Disorders. Journal of Autism Developmental Disorders, v. 44, p. 2185-2196, 2014.

BRITO, A. P. L.; NETO, A. R.; AMARAL, L. T. Síndrome de Asperger: Revisão de Literatura. Revista de Medicina e Saúde de Brasília, Brasília, v. 2, n. 3, p. 169-176, 2013.

CAMARGO, S. P. H.; RISPOLI, M. Análise do Comportamento Aplicada como intervenção para o autismo: definição, características e pressupostos filosóficos. Revista Educação Especial, Santa Maria, v. 26, n. 47, p. 639-650, 2013.

CAMARGOS JR, W. Semiologia clínica da Síndrome de Asperger. In: CAMARGOS JR, W. Síndrome de Asperger e outros Transtornos do Espectro do Autismo de Alto Funcionamento: da avaliação ao tratamento. Belo Horizonte: Artesã, p. 41-70, 2013.

CAMARGOS JR, W; TEIXEIRA, I. A. Síndrome de Asperger em mulheres. In: CAMARGOS JR., W. Síndrome de Asperger e outros Transtornos do Espectro do Autismo de Alto Funcionamento: da avaliação ao tratamento. Belo Horizonte: Artesã, p. 87106, 2013.

CENTERS FOR DISEASE CONTROL AND PREVENTION. Changes in Prevalence of Parent-reported Autism Spectrum Disorder in School-aged U.S. Children: 2007 to 20112012, 2013. Disponível em: http://www.cdc.gov/nchs/data/nhsr/nhsr065.pdf. Data de acesso: jul. 2017.

DANTAS, T. C.; SILVA, J. S. S.; CARVALHO, M. E. P. Entrelace entre gênero, sexualidade e deficiência: uma história feminina de rupturas e empoderamento. Revista Brasileira de Educação Especial, Marília, v. 20, n. 4, p. 555-568, 2014.

DEKKER, L. P. et al. Improving Psychosexual Knowledge in Adolescents with Autism Spectrum Disorder: Pilot of the Tackling Teenage Training Program. Journal of Autism Spectrum Disorders, v. 45, 1532-1540, 2015.

FISHER, M. H.; MOSKOWITZ, A. I.; HODAPP, R. M. Differences in social vunerability among individuals with autism spectrum disorder, Williams syndrome, and Down syndrome. Research in Autism Spectrum Disorders, v. 7, p. 931-937, 2013. 
FRANÇA-RIBEIRO, H. C. F. Direitos sexuais e pessoas com deficiência: conquistas e impasses. In: RIBEIRO, P. R. M.; FIGUEIRÓ, M. N. D. Sexualidade, cultura e educação sexual: propostas para reflexão. São Paulo: Cultura Acadêmica, p. 9-65, 2004.

GILMOUR, L.; SCHALOMON, P. M.; SMITH, V. Sexuality in a comumunity based sample of adults with autism spectrum disorder. Research in Autism Spectrum Disorders, v. 6, p. 313-318, 2012.

HELLEMANS, H.; COLSON, K.; VERBRAEKEN, C.; VERMEIREN, R.; DEBOUTTE, D. Sexual Behavior in High-Functioning Male Adolescents and Young Adults with Autism Spectrum Disorder. Journal of Autism and Developmental Disorders, v. 37, p. 260-269, 2007.

KALYVA, E. Teacher's perspectives of the sexuality of children with autismo spectrum disorders. Research in Autism Spectrum Disorders. V. 4, p. 433-437, 2010.

KLIN, A. Autismo e Síndrome de Asperger: uma visão geral. Revista Brasileira de Psiquiatria, v. 28, p. S3-S11, 2006.

KOLLER, R. Sexuality and Adolescents with Autism. Sexuality and Disability, v. 18, n. 2, p. 125-135, 2000.

LAMPREIA, C. Algumas considerações sobre a identificação precoce do autismo. In: MENDES, E. G.; ALMEIDA, M. A.; HAYASHI, M. C. P. Temas em educação especial: conhecimentos para fundamentar a prática. Araraquara: Junqueira \& Marin; Brasília: CAPESPROESP, p. 397-421, 2008.

MAIA, A. C. B. Educação sexual de pessoas com deficiência mental. In: FIGUEIRÓ, M. N. D.; RIBEIRO, P. R. M.; MELO, S. M. M. (Org.) Educação sexual no Brasil: panorama de pesquisas do sul e do sudeste. São Paulo: Cultura Acadêmica, p. 141-148, 2009a.

MAIA, A. C. B. Sexualidade, Deficiência e Gênero: reflexões sobre padrões definidores de normalidade. In: JUNQUEIRA, R. D. (Org). Diversidade Sexual na Educação: problematizações sobre homofobia nas escolas. Brasília: UNESCO, p. 265-291. 2009b.

MAIA, A. C. B. Inclusão e Sexualidade: na voz de pessoas com deficiência física. Curitiba: Juruá, 2011.

MAIA, A. C. B.; REIS-YAMAUTI, V. L.; SCHIAVO, R. A.; CAPELLINI, V. L. M. F.;

VALLE, T. G. M. Opinião de professores sobre a sexualidade e a educação sexual de alunos com deficiência intelectual. Estudos de Psicologia, Campinas, v. 32, n. 3, p. 427-435, 2015.

MAIA, A. C. B.; RIBEIRO, P. R. M. Desfazendo mitos para minimizar o preconceito sobre a sexualidade de pessoas com deficiência. Revista Educação Especial, Marília, v. 16, n. 2, p. 159-176, 2010.

MAHONEY, A.; POLING, A. Sexual abuse Prevention for People With Severe Developmental Disabilities. Journal of Developmental Disabilities, v. 23, p. 369-376, 2011.

MEHZABIN, P; STOKES, M. A. Self-assessed sexuality in young adults with HighFunctioning Autism. Research in Autism Spectrum Disorders, v. 5, 614-621, 2011. 
MOREIRA, L. M. A. Algumas abordagens da educação sexual na deficiência mental. Salvador: EDUFBA, 2007.

NATALE, L. L.; OLIVEIRA, L. F. S. Aspectos da sexualidade das pessoas com Transtornos do Espectro do Autismo de Alto Funcionamento. In: CAMARGOS JR., W. (Org.) Síndrome de Asperger e outros Transtornos do Espectro do Autismo de Alto Funcionamento: da avaliação ao tratamento. Belo Horizonte: Artesã, p. 213-228, 2013.

NEWPORT, J.; NEWPORT, M. Autism-Asperger's \& sexuality - puberty and beyond. Arlington, Texas: Future Horizons, 2002.

OUSLEY, O. Y.; MESIBOV, G. B. Sexual Attitudes and Knowledge of High-Functioning Adolescents and with Autism. Journal of Autism and Developmental Disorders, v. 21, n. 4, 471-481, 1991.

ROSQVIST, H. B. Becoming na "Autistic Couple": Narratives of Sexuality and Couplehood Within the Swedish Autistic Self-advocacy Movement. Sexuality and Disability, v. 32, 351$363,2014$.

SEGAR, M. Guia de sobrevivência para portadores de Síndrome de Asperger, 1997.

Disponível em: http://atividadeparaeducacaoespecial.com/wpcontent/uploads/2014/08/GUIA-DE-SOBREVIVENCIA-DA-SINDROME-DEASPERGER.pdf. Data de acesso: jul. 2017.

SEVLEVER, M.; ROTH, M. E.; GILLIS, J. M. Sexual Abuse and Offending in Autism Spectrum Disorders. Sexuality and Disability, v. 31, p. 189-200, 2013.

SMEHA, L. N.; CEZAR, P. K. A vivência da maternidade de mães de crianças com autismo. Psicologia em Estudo, Maringá, v. 16, n. 1, p. 43-50, 2011.

STOKES, M. A.; KAUR, A. High-functioning autim and sexuality. The National Autistic Society, v. 9, n. 3, p. 266-289, 2005.

STOKES, M.; NEWTON, N.; KAUR, A. Stalking, and Social, and Romantic Functioning Among Adolescents and Adults with Autism Spectrum Disorder. Journal of Autism and Developmental Disorders, v. 37, p. 1969-1986, 2007.

TISSOT, C. Establishing a sexual identity: Case studies of learners with autism and learning difficulties. Autism Journal of Sage Publications, v. 13. p. 551-566, 2009.

TARNAI, B.; WOLFE, P. S. Social Stories for Sexuality Education for Persons with Autism/Pervasive Developmental Disorder. Sexuality and Disability, v. 26, p. 29-36, 2008.

TEODORO, M. C.; CASARINI, K. A.; SCORSOLINI-COMIN, F. Intervenções Terapêuticas em pessoas com Síndrome de Asperger: uma revisão de literatura. Barbarói, Santa Cruz do Sul, n. 38, p. 6-25, 2013.

VIEIRA, A. C. Sexualidade e Transtorno do Espectro Autista: relatos de familiares. 2016. 164 f. Dissertação (Mestrado em Psicologia). Universidade Estadual Paulista "Júlio de Mesquita Filho", Faculdade de Ciências. Bauru, 2016. 
VIEIRA, A. C.; MAIA, A. C. B. Educação Sexual para Alunos com Deficiência na Escola Inclusiva: Análise sobre o Relato de Educadores. In: V Congresso Brasileiro de Educação, 2013. Anais [...] do V Congresso Brasileiro de Educação. Disponível em:

http://www2.fc.unesp.br/cbe/anais_iv-cbe.pdf. Data de acesso: jul. 2017.

\section{Como citar este artigo}

OTTONI, Ana Carla Vieira; MAIA, Ana Claudia Bortolozzi. Considerações sobre a sexualidade e educação sexual de pessoas com Transtorno do Espectro Autista. Revista IberoAmericana de Estudos em Educação, Araraquara, v. 14, n. esp. 2, p. 1265-1283, jul. 2019. E-ISSN: 1982-5587. DOI: 10.21723/riaee.v14iesp.2.12575

Submetido em: 30/09/2018

Revisões requeridas: 20/02/2019

Aprovado em: 30/04/2019

Publicado em: 25/06/2019 